\title{
The influence of initial geometric imperfections on the buckling load of single and double curvature concrete shells
}

\author{
Antonio Tomás*, Juan Pedro Tovar \\ Department of Civil Engineering, Universidad Politécnica de Cartagena, Paseo Alfonso XIII, 50, 30203 Cartagena (Murcia), Spain
}

\section{A R T I C L E I N F O}

\section{Article history:}

Received 5 November 2011

Accepted 11 January 2012

Available online 4 February 2012

\section{Keywords:}

Imperfection sensitivity factor

Buckling

Concrete shells

\begin{abstract}
A B S T R A C T
Several traditional methods are available to address the problem of instability in concrete shells, usually by means of curves representing the influence of the initial geometric imperfections in the buckling load for simple geometries (spheres and cylinders). Although some revisions of these methods have been made, new curves for other geometries have not been stated. In this study, the imperfection sensitivity factor in shell structures with different geometries (spherical dome, barrel vault, and double-curvature ruled surface) is determined graphically. This graphical method is useful to the designer in the early stages of the design of shell structures when dimensioning.
\end{abstract}

(c) 2012 Elsevier Ltd. All rights reserved.

\section{Introduction}

The buckling load of shells constructed in homogeneous elastic material sharply decreases with increasing initial imperfection amplitude $w_{0}$. This decrease is due to the magnitude of the imperfection itself and the eccentricity $e_{0}$ of the compressive force caused by this imperfection. In the case of homogeneous material shells, the stiffness of the shell cross section is practically independent of the eccentricity, so it is sufficient to investigate only the decrease of the buckling load with increasing imperfection alone. However, the plastic deformation; the load bearing capacity provided by the shell wall; and the stiffness of the cracked reinforced concrete cross section are heavily dependent on the eccentricity of the normal force applied. The influence of the imperfection $w_{0}$ and that of the eccentricity $e_{0}$ of the normal force may be dealt with separately. However, a relationship between both may be stated [1].

In the late 1970s, the International Association for Shell and Spatial Structures (IASS) Working Group No. 5 [2] devised a document of recommendations for reinforced concrete shells. The section on stability analysis includes a procedure based on the results of some previous research, particularly on the work of Kollár and Dulácska [3]. The procedure involves several factors that affect the linear buckling load to take into account concrete as an inhomogeneous nonlinear material. The design buckling load $p_{d}$ is obtained by applying these factors to the bifurcational load

$\gamma p_{\mathrm{d}}=\rho_{\mathrm{hom}} \alpha_{1} \alpha_{2} \alpha_{3} p_{\mathrm{cr}}$

\footnotetext{
* Corresponding author. Tel./fax: +34 968325653.

E-mail address: antonio.tomas@upct.es (A. Tomás).
}

where $\gamma$ is the factor of safety, $p_{\mathrm{cr}}$ the bifurcational or linear buckling load, $\rho_{\text {hom }}$ the imperfection sensitivity factor, $\alpha_{1}$ the creep factor, $\alpha_{2}$ the cracking and reinforcement factor, and $\alpha_{3}$ the inelasticity of concrete factor.

Scordelis [4] and Medwadowski [5] observed that the method provides conservative results in the case of spherical domes. However, Kollár [6] compared the theoretical predictions with experimental results and found great similarity. The first review of the Recommendations was prepared by Kollár [7]. Years later, Medwadowski [5] went back to revise the initial and the Kollár proposals, presenting some modifications and suggestions.

The geometric imperfection sensitivity factor is the relationship of the upper critical load (the buckling load calculated by a geometric nonlinear analysis) with respect to the buckling critical load for linear homogeneous material (calculated by a linear or eigenvalue analysis, and proves to be greater than the upper critical load in most cases). The calculation of this factor is often difficult. Some cases appear in the technical literature, such as spheres and cylinders [2]. These analytical solutions have the advantage of using abacuses and simple formulas to obtain the buckling load, but have the disadvantage of predicting this type of behaviour only for certain theoretical cases (sphere and cylinder), not being able to deal with structures with other geometries. Some differences between analytical and numerical solutions for an actual shell can be consulted in $[8,9]$.

In the absence of further information about the geometry, the IASS recommends using the safest case (sphere under radial pressure and axially compressed cylinder). However, in most cases this approach is too conservative. Thus, it may be useful to study new geometries to obtain a better approach to their structural behaviour against imperfection. 
This paper is an updated and revised version of the conference paper [10]. In said paper, a method based on that used in $[1,3,11]$ is implemented to graphically determine the imperfection sensitivity factor in the case of shells of different geometries (such as spherical dome, barrel vault, and double-curvature ruled: hyperbolic paraboloid and hyperbolic rotational surface) to those studied in the IASS Recommendations.

Although admitting that the graphical methods in structural mechanics are at present being replaced by numerical methods, the former are useful to the designer in the early stages of the design of shell structures at the level of dimensioning. Moreover, from the academic point of view, the graphical methods are particularly useful in the teaching of structural engineering.

In the present study, the contents of Tomás et al. [10] have been revised and expanded, namely: details are included of several proposals to quantify the initial geometric imperfection; the number of references has been increased; and application to different cases has been achieved by providing three detailed examples of outstanding shell structures in Spain. These examples are the dome of Algeciras market; the cooling tower of the Ascó nuclear power plant; and the access building to L'Oceanogràific (the Oceanographic Park at Valencia).

\section{Imperfection sensitivity factor for homogeneous elastic material}

The value of this factor is one if the shell is not sensitive to imperfection, and is otherwise less than one. The initial geometric imperfection sensitivity factor $\rho_{\text {hom }}$ is the relationship of the upper critical load $p_{\mathrm{cr}}^{\text {upper }}$ with respect to the buckling critical load for linear homogeneous material $p_{\mathrm{cr}}^{\text {lin }}$.

The calculation of $\rho_{\text {hom }}$ is often difficult. Some cases may be found in technical literature. Fig. 1 shows the variation of $\rho_{\text {hom }}$ with respect to $w_{0} / e$, where $w_{0}$ is a measure of the imperfection and $e$ is the thickness of the shell. The curves A, B and $\mathrm{C}$ belong to laterally compressed long $\left(L^{2} / R e=10000\right)$, medium $\left(L^{2} /\right.$ $R e=1000)$, and short $\left(L^{2} / R e=100\right)$ cylinders, respectively, with $L$ length and $R$ radius. The curve $\mathrm{D}$ is for spheres and axially compressed cylinders.

The initial imperfection consists of accidental imperfection $w_{0}^{\prime \prime}$, due to erection inaccuracies, and calculable imperfection $w_{0}^{\prime}$, quantified by the bending theory of shells (a value of maximum

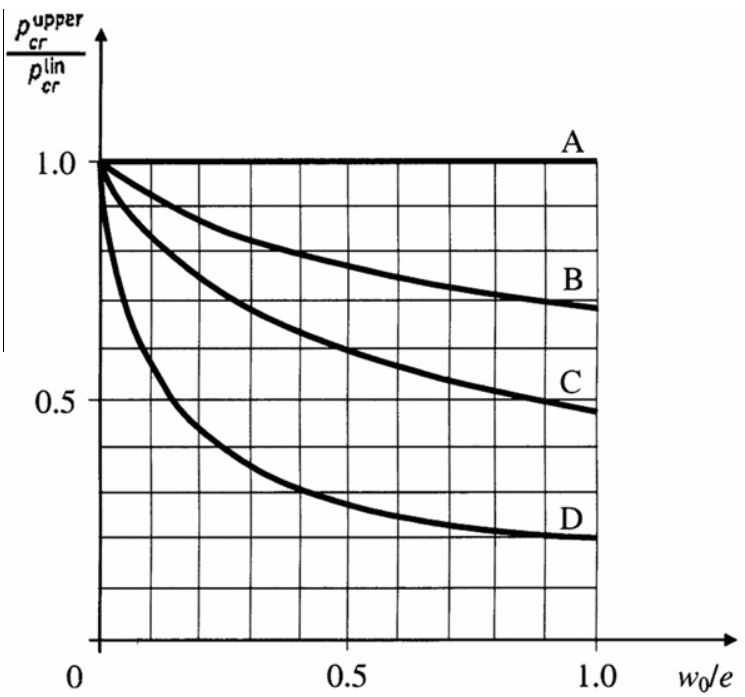

Fig. 1. Initial geometric imperfection sensitivity factor $\left(\rho_{\text {hom }}=p_{\mathrm{cr}}^{\text {upper }} / p_{\mathrm{cr}}^{\text {lin }}\right)$ in concrete shells, using concrete as homogeneous material [2]. deflection obtained for a certain service load combination using a linear elastic analysis)

The maximum values of both imperfections are unlikely to coincide. According to the probability theory, Kollár and Dulácska [1] suggested taking the higher of the following values to choose the initial geometric imperfections $w_{0}$

$w_{0} \geqslant\left\{\begin{array}{c}w_{0}^{\prime}+0,8 w_{0}^{\prime \prime} \\ w_{0}^{\prime \prime}\end{array}\right\}$

Small deviations that may occur when estimating this value are considered by using a safety factor. In the case of preferring a more conservative way of designing, both types of imperfections may be taken into account at the same time.

To obtain $w_{0}^{\prime \prime}$, Kollár and Dulácska [1,3] proposed the following expression

$w_{0}^{\prime \prime} \approx \frac{R}{3500}$

where $R=\sqrt{R_{1} R_{2}}$ if the shell is double curvature, with $R_{1}$ and $R_{2}$ being the mean principal radii of curvature of the shell. In the case of a sphere, both radii have the same value.

Eq. (3) is an approximate expression to quantify the accidental imperfection of a shell. This equation was used to construct the curves of Fig. 1. Using this approach and modelling the shell by finite elements, the influence of the accidental imperfection may be stated by means of a geometric nonlinear analysis, which takes into account the effect of large displacements. However, it must be borne in mind that this equation is only valid for carefully erected shells (by using rigid formwork) and for a thickness between 0.05 and $0.07 \mathrm{~m}$.

By the method of the IASS Recommendations, the linear buckling load of the perfect homogeneous shell $p_{\mathrm{cr}}^{\mathrm{lin}}$ is used as a starting point. This load must be calculated accurately, preferably by a FEM linear elastic analysis. The effect of large deformations is considered by the maximum bending deflection of the shell $w_{0}^{\prime}$, which may be estimated numerically or experimentally. Then, with the ratio deflection/thickness $\left(w_{0}^{\prime} / e\right)$ and Fig. 1 , the upper critical load $p_{\mathrm{cr}}^{\text {upper }}$ for homogeneous elastic material is obtained. If the geometry of the shell does not match any of the curves shown in Fig. 1, and in the absence of further information, the curve $D$ for spheres and axially compressed cylinders must be used.

Since the geometry of the actual shell differs from the theoretical design, if the post-buckling effect is important (phenomenon present at shells used in building construction), the IASS Recommendations propose to reduce the linear critical load in a similar way, i.e., estimating the deviation $w_{0}^{\prime \prime}$ on the surface and using the ratio $w_{0}^{\prime \prime} / e$ and Fig. 1.

If both effects are present simultaneously, the IASS Recommendations propose that the reduction procedure must be performed at the same time using the geometric imperfection as $w_{0}=w_{0}^{\prime}+w_{0}^{\prime \prime}$.

In 1993, Kollár [7] proposed the following equation to quantify the initial geometric imperfections in shells

$w_{0}=\sqrt{\left(w_{0}^{\prime}\right)^{2}+1.4 w_{0}^{\prime} w_{0}^{\prime \prime}+\left(w_{0}^{\prime \prime}\right)^{2}}$

The form of this equation reflects the low probability that the largest values of $w_{0}^{\prime}$ and $w_{0}^{\prime \prime}$ occur at the same location on the shell. In the same proposal [7], the value of $w_{0}^{\prime \prime}$ is to be calculated from the empirical equation

$w_{0}^{\prime \prime}=0.05 e+\frac{e a}{2\left(1+\beta^{-2}\right)}$

where $\beta=0.001(R / e)$ and the coefficient $a$ is intended to account for the type of formwork, with $a=1$ for rigid forms, and $a=6$ for slipforms. 
Thus, the value of $w_{0}^{\prime \prime}$ varies between the practical limits of $w_{0}^{\prime \prime}=0.05 e$ (thick shells) to $w_{0}^{\prime \prime}=0.3 e a$ (thin shells).

Medwadowski [5] considers that the empirical formula proposed by Kollár yields small values of imperfections for very thin shells. However, the equation contains no provision for the value of $R$ for shells of double curvature, other than spheres. For those reasons, the following modification is suggested

$w_{0}^{\prime \prime}=0.1 e\left[1+\frac{5 a}{\left(1+\beta^{-2}\right)}\right]$

where

$\beta=0.001 \sqrt{R_{1} R_{2}} / e$

where $R_{1}, R_{2}$ principal radii of curvature of the shell, $a$ as before, $a=1$ for a shell cast on a rigid formwork, and $a=6$ for a shell built using slipforms.

It can be extrapolated, observing Eq. (6), that the equations proposed by Kollár are also valid for the calculation of accidental imperfections in the case of double curvature shells, just by taking $R=\sqrt{R_{1} R_{2}}$.

\section{Imperfection sensitivity factor for several geometries of shells}

\subsection{Introduction}

The curves of the influence of geometric imperfection in the critical buckling load of shells have only been defined for certain geometrical models (spheres and cylinders). For this reason, in the absence of further information, the IASS recommends to use the safest curve (curve $D$ for a sphere under radial pressure and axially compressed cylinder). However, this approach in most cases is too conservative. Thus, it may be useful to obtain new curves for other geometries in order to have a better approximation of their structural behaviour against imperfection.

In this section, the curves for several geometrical models of single and double curvature are presented [12]. Previously, validation of the method is performed by obtaining the curves corresponding to spheres and cylinders and by comparing them with the curves of the IASS Recommendations.

\subsection{Sphere and cylinder. Validation of analysis method}

To study the behaviour of the models against accidental geometric imperfection $\left(w_{0}^{\prime \prime}\right)$, the critical buckling load for each design is calculated by a linear or eigenvalue analysis $\left(p_{\mathrm{cr}}^{\text {lin }}\right)$ and a geometric nonlinear analysis $\left(p_{\mathrm{cr}}^{\text {upper }}\right)$. Relating both results, the imperfection sensitivity factor $\left(\rho_{\text {hom }}\right)$ is obtained for a given value of $w_{0}\left(=w_{0}^{\prime \prime}\right)$.

Eq. (3) is used, as Kollár and Dulácska did [1,3], to quantify the accidental imperfection in the shell up to values of $w_{0} / e=1$ and higher, although this level has not been exceeded in this work in order to state a methodology similar to that in the IASS Recommendations. Each design was analysed for a thickness $e$ of 0.05 , 0.06 and $0.07 \mathrm{~m}$. These values are within the range of validity of Eq. (3).

That is, for a pair of values $(R, e), p_{\mathrm{cr}}^{\text {upper }}$ is obtained from a geometric nonlinear analysis and $p_{\mathrm{cr}}^{\text {lin }}$ is obtained from an eigenvalue analysis. $\rho_{\text {hom }}$ is achieved dividing $p_{\mathrm{cr}}^{\text {upper }}$ by $p_{\mathrm{cr}}^{\text {lin }}$. Therefore, a point of the curve is obtained, whose coordinates are $\left(w_{0} / e, \rho_{\text {hom }}\right)$. Logically, $R$ and $e$ have been chosen so that $w_{0} / e$ be set to the sequence $0.1,0.2, \ldots, 1.0$.

A tolerance of forces and displacements of $0.0001 \%$ has been used in the nonlinear analysis in order to obtain results with sufficient accuracy.
A mesh size that leads to a relative error less than or equal to $1 \%$ in the critical buckling load has been chosen. It does not lead to an excessively large computational cost and the obtained results have an acceptable error.

Different boundary conditions have been used (hinged edges, clamped edges, free edges but with a clamped support at the centre) in order to obtain a cloud of points with which to interpolate a curve. The influence of the boundary conditions in the model is not very crucial if the edges are not weaker than the shell itself, since most shells experience local buckling rather than global buckling [1]. As a priori buckling behaviour of each shell is unknown, all designs have been analysed with different restrictions (degrees of freedom) at their edges. Fig. 2 shows a graphical summary of $\rho_{\text {hom }}$ for several thicknesses and boundary conditions.

Using the validated method and following the same criteria stated in this section for the mesh size and the tolerance of forces and displacements, other usual geometrical configurations in the erection of shells are analysed.

\subsection{Barrel vault and cylindrical shell}

The influence of the imperfection is analysed for several boundary conditions to simulate different behaviour: barrel vault (supported on right edges) or cylindrical shell (supported on curve edges). The results are shown in Fig. 3.

\subsection{Spherical domes}

In this section, the imperfection sensitivity is analysed considering several types of configurations for a spherical dome. The configuration depends on the angle from the vertical plane with any line passing through the base of the dome and being perpendicular to the surface of it. Reduced domes are particularly interesting because their arches do not exceed the so-called neutral line, defined by the parallel between compressed parallels (upper cap) and tensioned parallels (lower cap). It is not usual to erect domes reduced more than an angle of $36^{\circ}$. Domes with an angle of $90^{\circ}, 60^{\circ}, 36^{\circ}$ and $20^{\circ}$ and a dome on a polygonal base with an angle of $36^{\circ}$ have been used in this study (Fig. 4).

Simple boundary conditions such as those which have been used (hinges and clamps) have little influence on results. However, there are sometimes cases where the critical load of spherical domes is reduced, not only because of imperfections, but also because of the boundary conditions on which they are supported [13].

\subsection{Hyperbolic rotational surface}

The study of the imperfection in these shells is complex because of being double-curved surfaces defined by two different radii. To estimate the imperfection sensitivity factor and the nonlinear buckling load in equidistant points $w_{0} / e$, it is necessary to state an average radius $\left(R_{\theta} R_{\phi}^{1 / 2}\right.$ that is obtained from two principal radii of curvature [5], which also depend on other geometrical parameters such as radius and the length of the top and the base. These principal radii of curvature vary along the rotation axis, so it is necessary to state a reference point to define them and be able to perform calculations. This kind of structures are usually characterized by the shape of their throat, which is considered as zero level, so it may be possible to refer the principal radii of curvature to this point. The calculation of these radii is developed in [12,14].

To state a common methodology to the cases considered in previous sections and to compare the results, it is necessary to study designs which meet the geometrical relationships of the short $\left(l^{2}\right)$ $R e=100)$, medium $\left(l^{2} / R e=1000\right)$ and long $\left(l^{2} / R e=10000\right)$ model. However, if the dimensions are forced to satisfy the short model, the form of the shell is distorted excessively. For this reason, only 

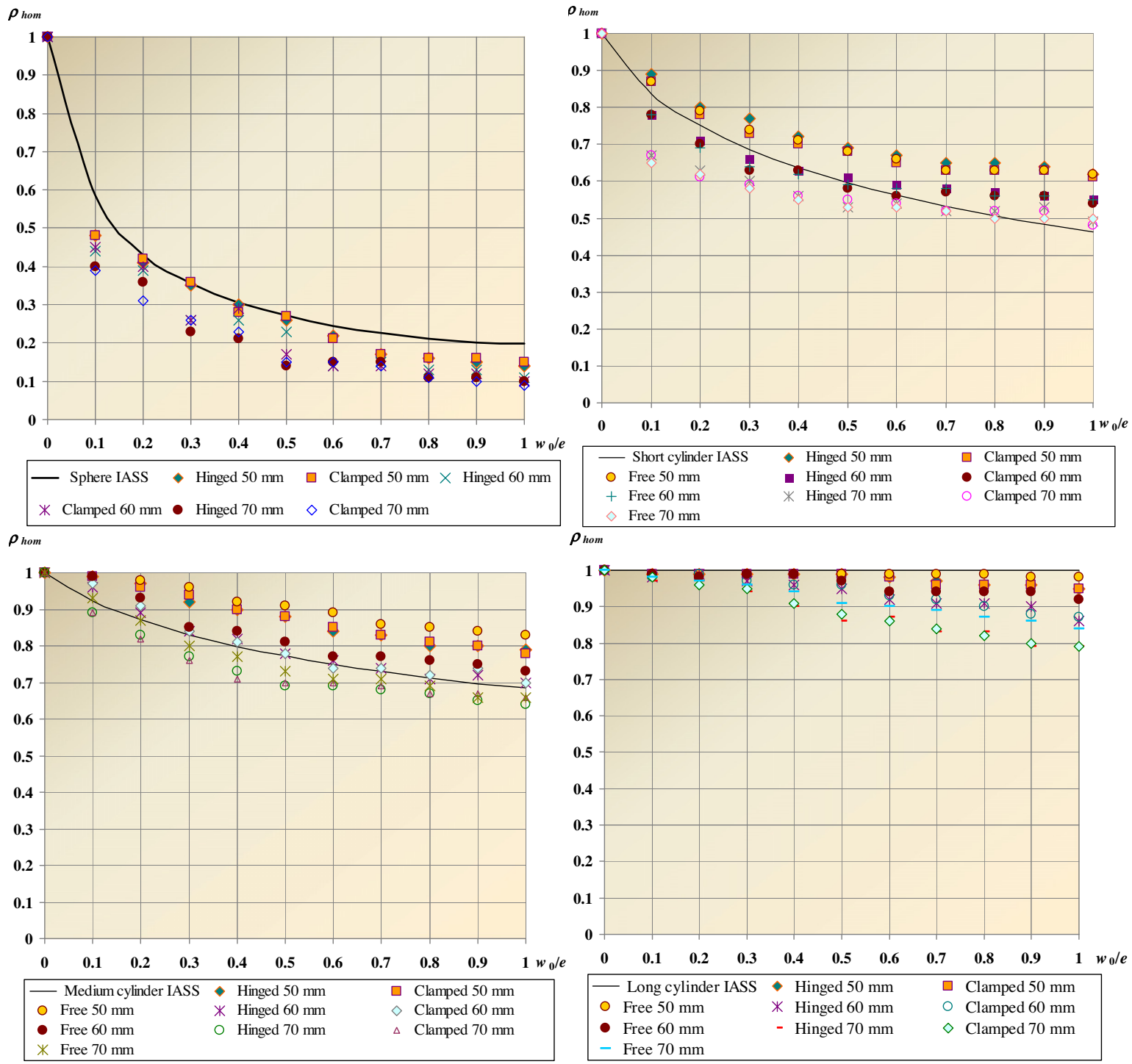

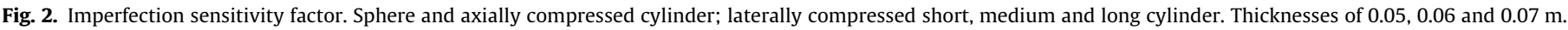
Hinged, clamped and free* supports. ( ${ }^{*}$ Free edges but with a clamped support at the centre.)

medium and long shells are considered, with several sizes, leading to equidistant values of the ratio $w_{0} / e$.

The most common structures with this type of geometry are cooling towers. The thickness of these towers is variable depending on their height. The expression $\left(\left(R^{2} H\right)^{1 / 3}\right) / 400$ may be used to determine the minimum thickness at different heights, where $R$ is the transverse radius of the tower and $H$ the height [15].

Initially, the medium and long hyperboloid designs are loaded to lateral compression, supported on two stiffness rings, which are hinged at both ends. The second case is the study of a hyperboloid between medium and long designs, subjected to axial load and supported on the same boundary conditions as above (Fig. 5a). This latter design keeps the same proportions as the actual cooling towers. This last geometry has been adopted since, as was the case with the cylinders, the differences shown in the results for axial load between the medium and long hyperboloid are very small.

The following case that has been studied is the revolution hyperboloid with clamped ends (Fig. 5b). It is not possible to study the shell subjected to axial load with these supports. The same models have also been analysed but with their ends free, supported on a stiffness ring in the mean cross section (Fig. 5c).
This type of geometrical configurations is used in the cooling towers of nuclear power plants. However, it is rare to find a cooling tower subjected to the loads and boundary conditions described previously. The case of axially compressed hyperboloid is perhaps closer to reality, but in no event with supports at the top. For this reason, it is advisable to perform an analysis to evaluate the imperfection sensitivity of a hyperboloid clamped on the base and subjected to gravitational load (Fig. 5d).

\subsection{Hyperbolic paraboloid (hypar)}

Two models have been defined by means of the translation of a reduced parabola and of a narrow parabola, both laterally and axially compressed.

As with rotational hyperboloid, the principal radii of curvature that define the geometries are referred to the throat of the models. These radii of curvature are different when moving from the throat transversally or longitudinally.

For this reason, the two above-mentioned designs need to be adopted, since two principal radii defined for any point of the shell do not take into account the shape of the parabola that defines the 
a

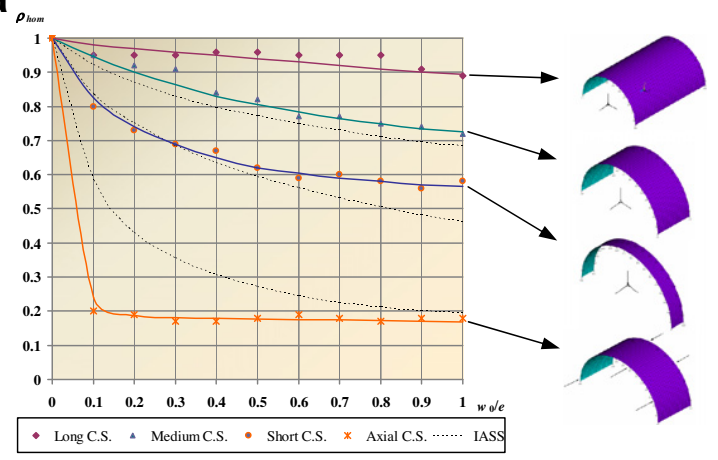

b

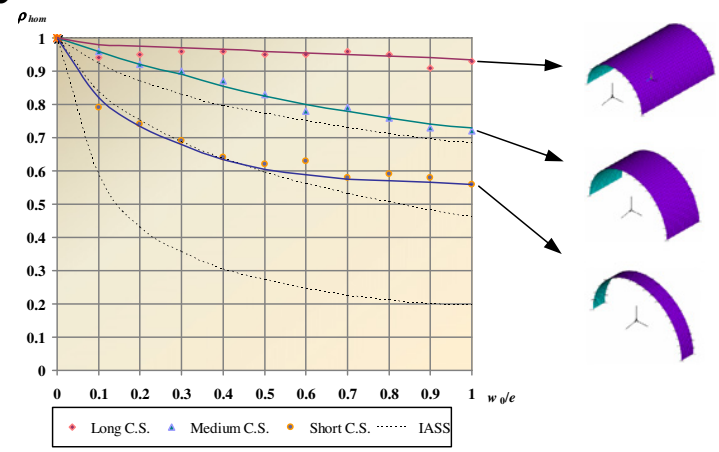

C

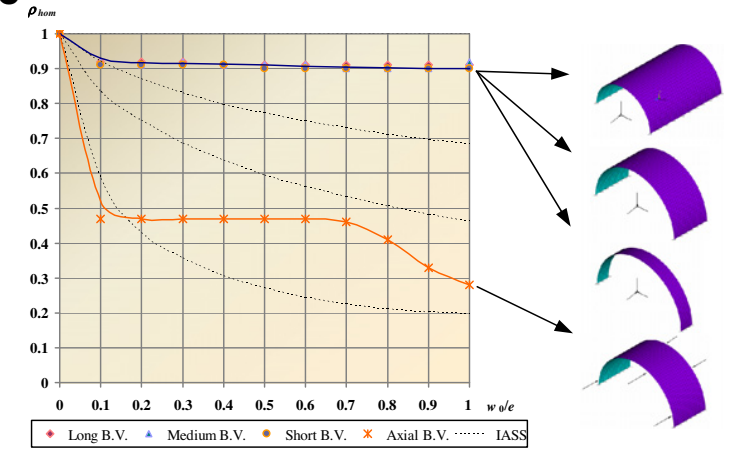

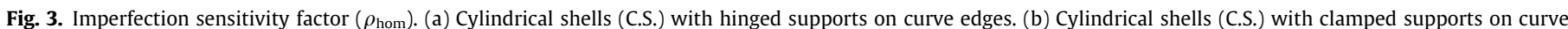

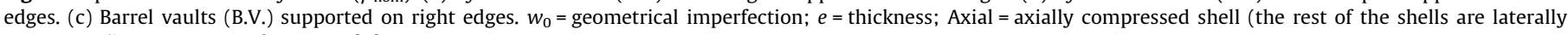
compressed); IASS = curves from IASS [2]

model. However, one of these radii considers the length and then it is not necessary to adopt several sizes of shells as was done in previous cases.

Several boundary conditions have been applied. Some of them have been used in previous geometries, and others are more appropriate for this particular case.

Fig. 6a contains the results of hypar hinged at both end edges, and Fig. $6 \mathrm{~b}$ and $\mathrm{c}$ of hypar clamped at both edges, lateral and end ones respectively. In the latter two cases, it is not possible to analyse the axial compression model because of the clamped ends. The hypar under lateral compression and axial load supported on the four clamped corners is shown in Fig. 6d. This support configuration is present in many real shells today.

\section{Examples}

\subsection{Introduction}

In this section, three examples of practical implementation are used: the access building to L'Oceanogràfic (The Oceanographic Park at Valencia), the dome of Algeciras Market, and the cooling tower of the Ascó nuclear power plant, all in Spain.

In these examples, the accidental imperfection sensitivity factor $\left(\rho_{\text {hom }}^{*}\right)$ was obtained graphically and analytically, and was compared with that obtained by the graph for spheres, as recommended by the IASS. Subsequently, the initial geometric imperfection sensitivity factor $\left(\rho_{\text {hom }}\right)$ is obtained in each example.

The case of $\rho_{\text {hom }}^{*}$ is simply the application of a particular case (with a given $R, e$ and certain boundary conditions) in order to obtain a point on the curve, both graphically and analytically, and to see that the difference is small, because the graph is an interpolated curve, while the numerical method is an exact value.
The case of $\rho_{\text {hom }}$ is to lead the application of the graphical method further, into a real case, considering both imperfections (the accidental one and the calculable one). These two imperfections are not added, as proposed by the IASS Recommendations, since the maximum values of both are very unlikely to coincide, but they should be used in combination in one of the equations proposed by several authors; Eq. (4) is one such example.

\subsection{L'Oceanogràfic entrance building, Valencia}

\subsubsection{The hyperbolic paraboloid (hypar)}

The calculation of the imperfection sensitivity factor of the concrete shell structure that is the access building to L'Oceanogràfic the posthumous work of Félix Candela - is presented below (Fig. 7). A complete description of the parameters and design variables used in the definition of the geometry may be consulted in $[8,16]$.

The analysis under predominant gravitational loads has been carried out, so it is only necessary to consider the specific weight of material $25 \mathrm{kN} / \mathrm{m}^{3}$ (a value commonly used for reinforced concrete). The loads are expressed as a multiplier of the weight. The thickness of the shell is $0.06 \mathrm{~m}$.

The shell has been stiffened by two types of ribs with similar dimensions as those used by Domingo et al. [17]. One type spreads from the support to the centre of the structure (main ribs). The other surrounds a small central hole made in the shell gauging a band of $0.20 \mathrm{~m}$ wide (hole rib). It was necessary to create that small circular hole of $0.10 \mathrm{~m}$ diameter at the intersection of the lobes, in order to avoid meshing problems arising from the distortion of the elements generated in the area surrounding the centre, which have very acute angles. 
a

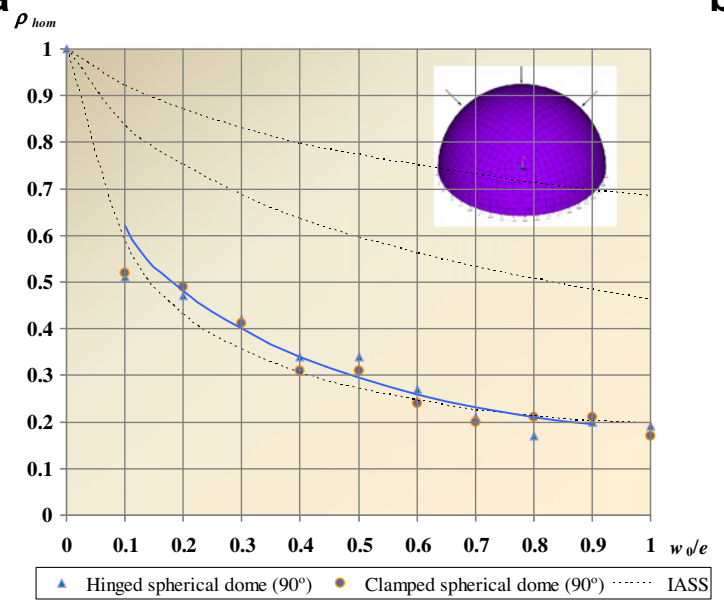

b

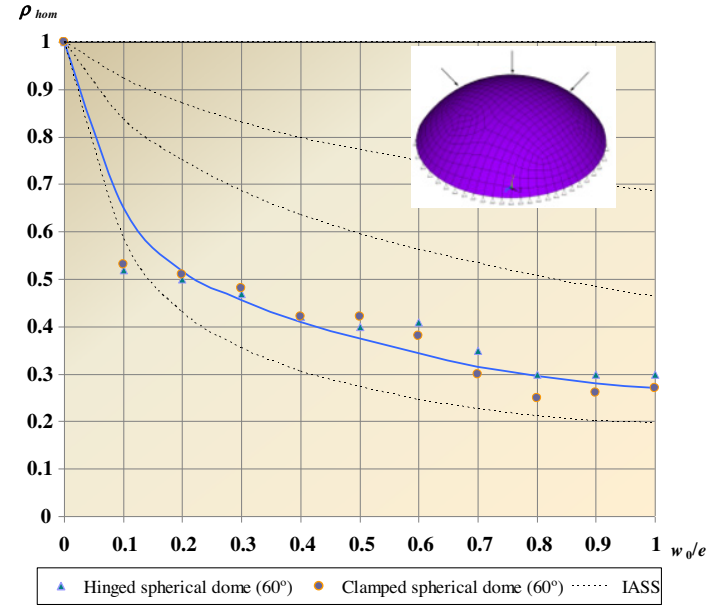

d

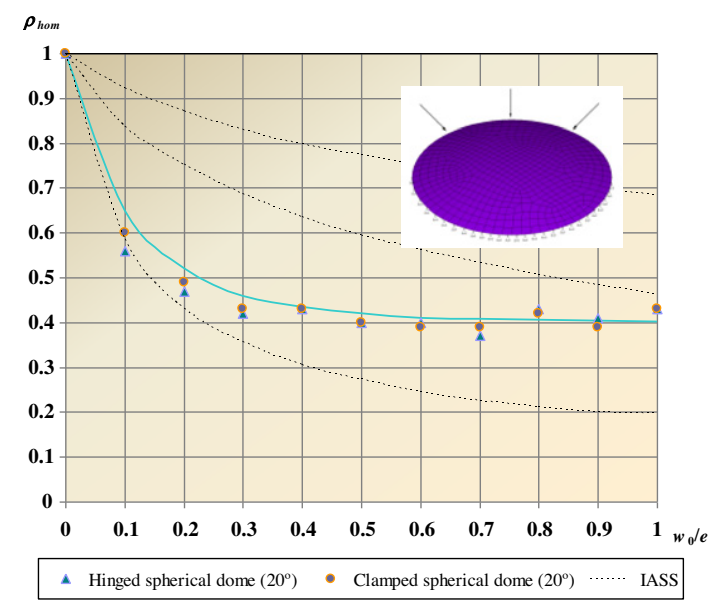

C

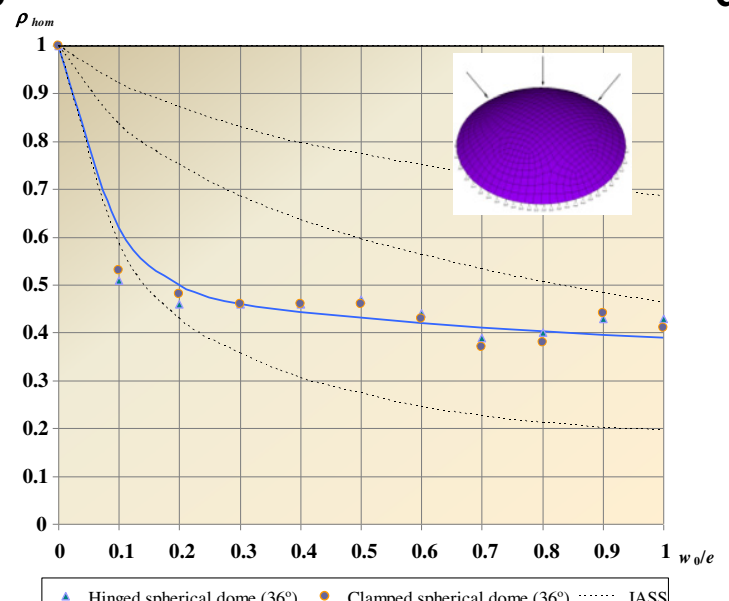



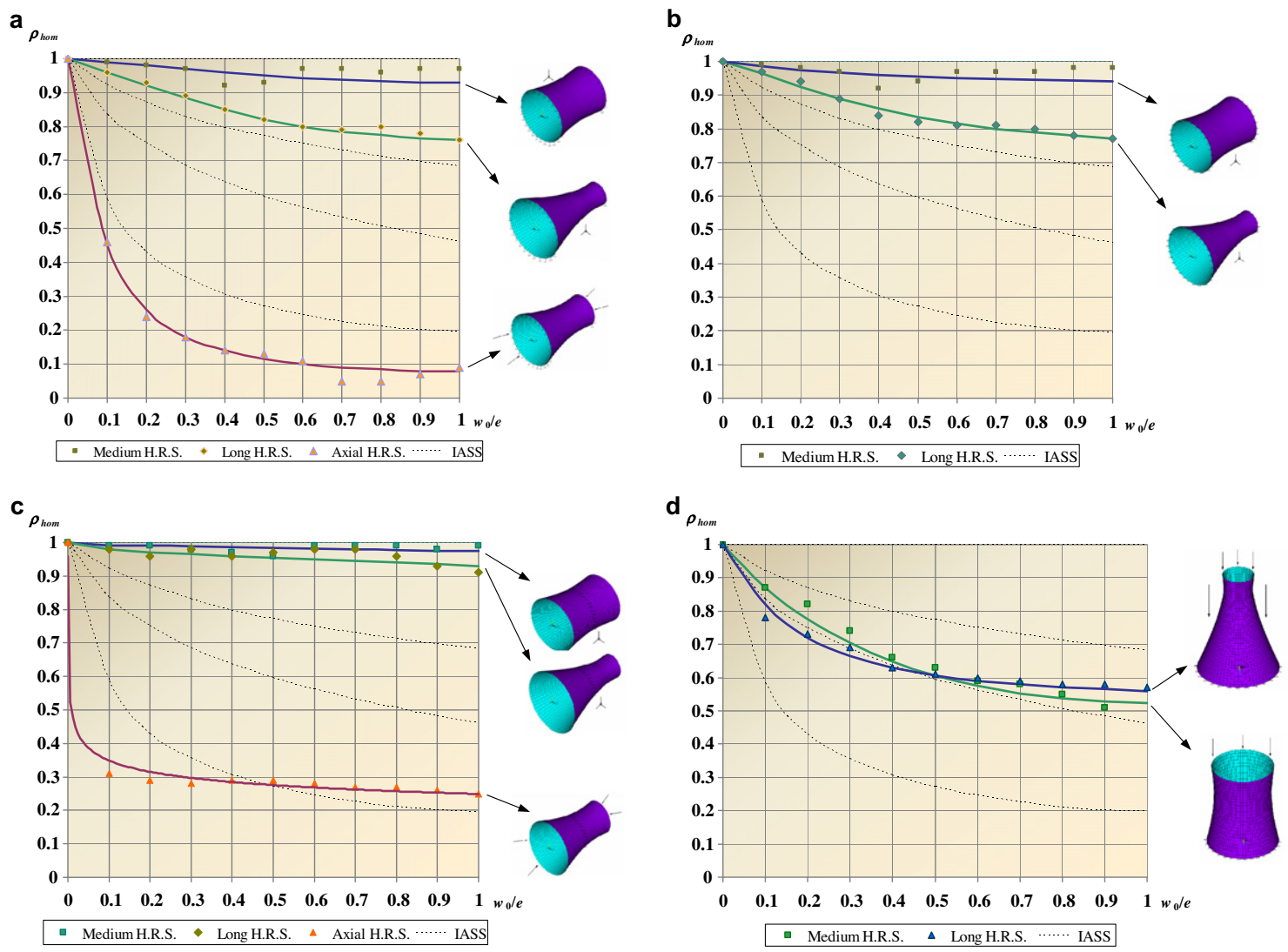

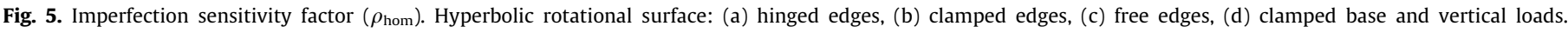

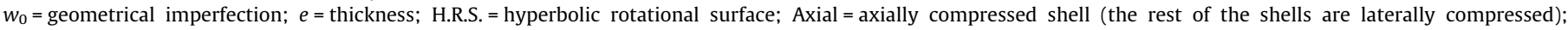
IASS $=$ curves from IASS [2]

noted that these curves are for laterally compressed shells, which are more conservative than in reality (shells are subjected under mainly gravitational load).

Among the calculation methods outlined in Section 2 (IASS, Kollár and Medwadowski) it is more appropriate to use Medwadowski's method, as particularized for double curvature shells.

To quantify the value of accidental imperfection it is necessary to know the value of the geometric parameters that define the structure: height at its centre $c=6.50 \mathrm{~m}$, height of the free edge of the lobe $H_{\mathrm{b}}=24.39 \mathrm{~m}$, radius or distance in plan from the centre of the shell to the support $D=13.63 \mathrm{~m}$. From the geometric definition it is possible to obtain the approximate principal radii of curvature [12] $\left(R_{\phi}=2.19 \mathrm{~m}\right.$ and $\left.R_{\theta}=6.50 \mathrm{~m}\right)$ giving rise to the average radius $R=3.77 \mathrm{~m}$ and the parameter $\beta=0.063$. Considering rigid formwork $(a=1), w_{0}^{\prime \prime}$ results $0.00612 \mathrm{~m}$ and the ratio $w_{0}^{\prime \prime} / e$ is 0.102 .

Using the graphs mentioned above, the accidental imperfection sensitivity factor $\rho_{\text {hom }}^{*}$ is obtained. The results for clamped lateral edges and clamped supports at corners are 0.78 and 1.00 respectively, with the average value of 0.89 (these values of $\rho_{\text {hom }}^{*}$ are expressed as multiplier of the weight).

In the event of not having these graphs for hypar shells, the curve for the sphere and the axially compressed cylinder would have been used, following the IASS Recommendations, so a value of 0.59 would have been obtained, which is considerably more conservative (33.71\% lower).

\subsubsection{Accidental imperfection sensitivity factor $\rho_{\mathrm{hom}}^{*}$. Analytical method}

To calculate the influence of accidental geometric imperfections analytically, it is necessary to carry out a buckling analysis of the structure. The ANSYS program of finite elements was used to model the shell [18].

Firstly, an eigenvalue (linear) analysis was carried out to calculate the linear buckling load $\left(p_{\mathrm{cr}}^{\text {lin }}=8.649\right)$. Then, a nonlinear analysis considering geometric and material nonlinearity was carried out. Since the behaviour of this type of shells is due to the form, only the concrete is modelled as material of the shell. The result was $p_{\mathrm{cr}}^{\text {upper }}=7.870$, lower than $p_{\mathrm{cr}}^{\text {lin }}$ because of the sensitivity of the shell to imperfections. Each value of the buckling load is shown as a factor of weight of the shell.

Dividing the two results, the accidental imperfection sensitivity factor is obtained $\rho_{\text {hom }}^{*}=p_{\mathrm{cr}}^{\text {upper }} / p_{\mathrm{cr}}^{\text {lin }}=0.91$.

Using the graphical method, the factor is $2.2 \%$ more conservative than the analytical result. This error is negligible given that $\rho_{\text {hom }}^{*}$ is corrected by a safety factor, which considers small deviations in the calculation. However, using the IASS Recommendations for spheres, the factor is $35.16 \%$ lower, resulting in an excessive over-sizing of the structure.

\subsubsection{Initial geometric imperfection sensitivity factor $\rho_{\text {hom }}$}

The calculable imperfection $w_{0}^{\prime}$ is quantified by the bending theory of shells (a value of maximum deflection obtained for a certain service load combination using a linear elastic analysis).

In this research, it is necessary to analyse the structure to obtain the deflection just before the start of buckling. In a real case, if the aim is to obtain a value of $\rho_{\text {hom }}$ for a shell with a certain geometry, without comparing it with that of the sphere, the designer must use the deflection of the shell starting with the worst service load combination. However, no load combination has been used in this research, but the highest possible deflection in the shell has been chosen, i.e., the deflection just before the start of buckling. It does 
$\mathbf{a}_{\rho_{1}}$

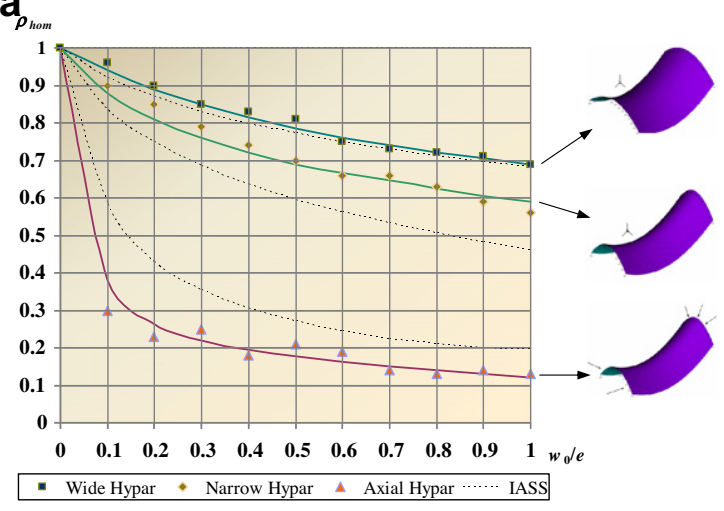

C

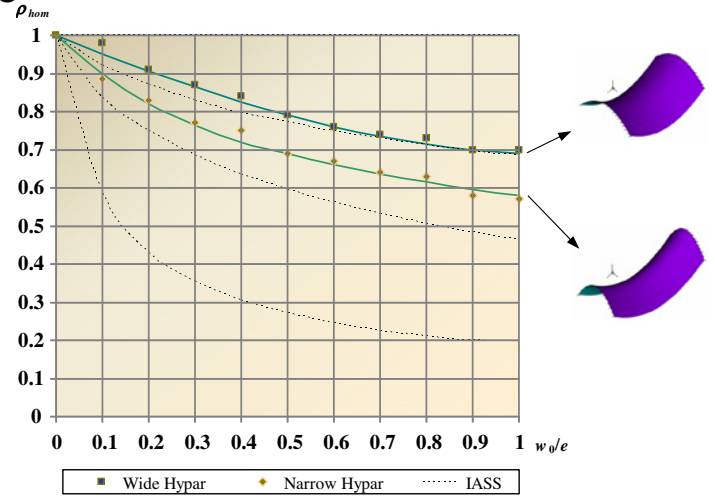

b

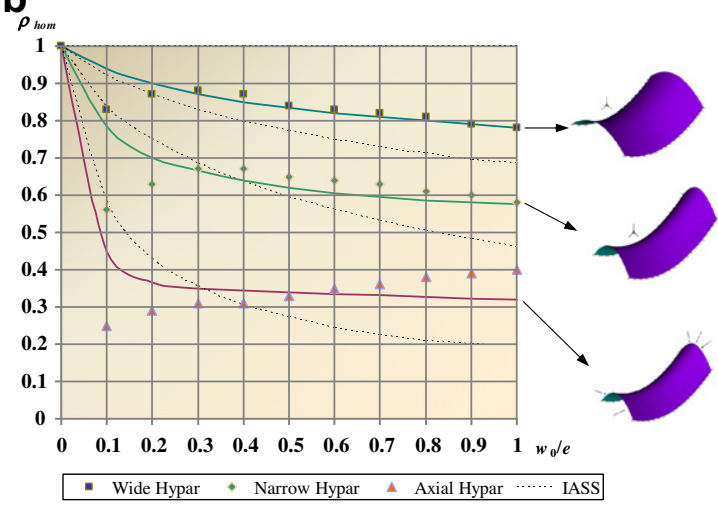

d

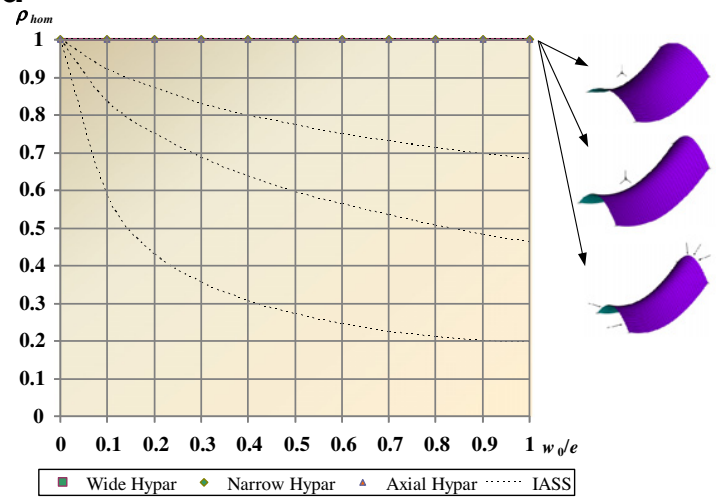

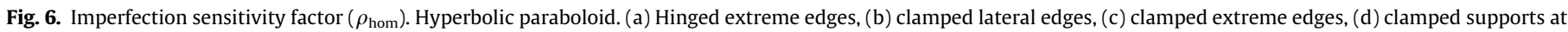

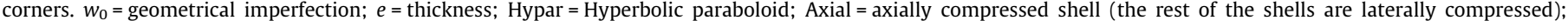
IASS = curves from IASS [2]
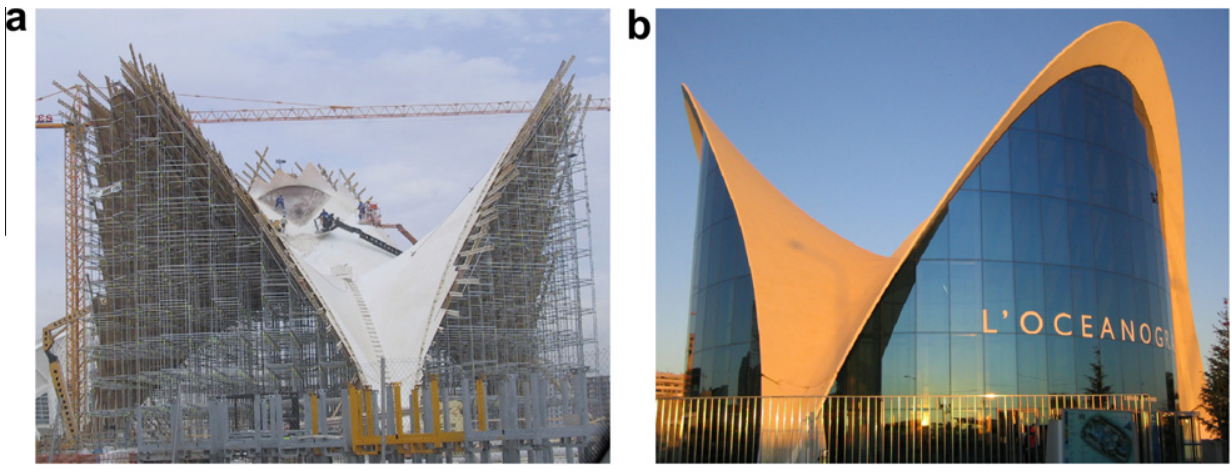

Fig. 7. Shell structure at the entrance of the L'Oceanogràfic at Valencia, Spain. (a) The shell under construction. (b) The shell today. [Photos by A. Tomás (2001, 2005)].

not matter which deflection is chosen if the aim is to graphically compare the $\rho_{\text {hom }}$ of a certain geometry with the $\rho_{\text {hom }}$ of the sphere, since the result is a ratio that relates both $\rho_{\text {hom }}$, a ratio that is independent on the chosen deflection.

Fig. 9 shows the displacements in the hypar and the deformed shell. The maximum displacement $w_{0}^{\prime}$ is $0.0546 \mathrm{~m}$.

Using Eq. (4), the initial global geometric imperfection of the shell $w_{0}$ is $0.0591 \mathrm{~m}$ and the ratio $w_{0} / e$ is 0.99 . Using graphs in Fig. $6 \mathrm{~b}$ and $d$, the average value of the initial geometric imperfection sensitivity factor $\rho_{\text {hom }}$ is 0.79 .

Again, there is a noticeable difference between this factor and that obtained using the curve for spheres. In the latter case, the factor is $0.20,74.68 \%$ lower than the result obtained with the curves of Fig. $6 \mathrm{~b}$ and $\mathrm{d}$.

It is appropriate to clarify why the deflection just before the start of buckling has been used to obtain $\rho_{\text {hom }}$ : because it is easy to achieve, and the resulting $\rho_{\text {hom }}$ is only used for comparison with the $\rho_{\text {hom }}$ of the sphere from the IASS Recommendations. That is to say that any value of the deflection is valid in order to draw a comparison. In a real case, if $\rho_{\text {hom }}$ is required to be used in the design (without being compared with the sphere value), the designer must use the deflection of the shell from the worst combination of service loads.

\subsection{Algeciras market}

\subsubsection{The dome}

The roof, designed by Eduardo Torroja, consists of a spherical cap of reinforced concrete with a radius of $44.20 \mathrm{~m}$ (Fig. 10a). The dome has a diameter of $47.60 \mathrm{~m}$ and a thickness of $0.09 \mathrm{~m}$. In the centre of the dome, a large octagonal skylight, with the main diagonal of $10 \mathrm{~m}$, provides the interior lighting. 


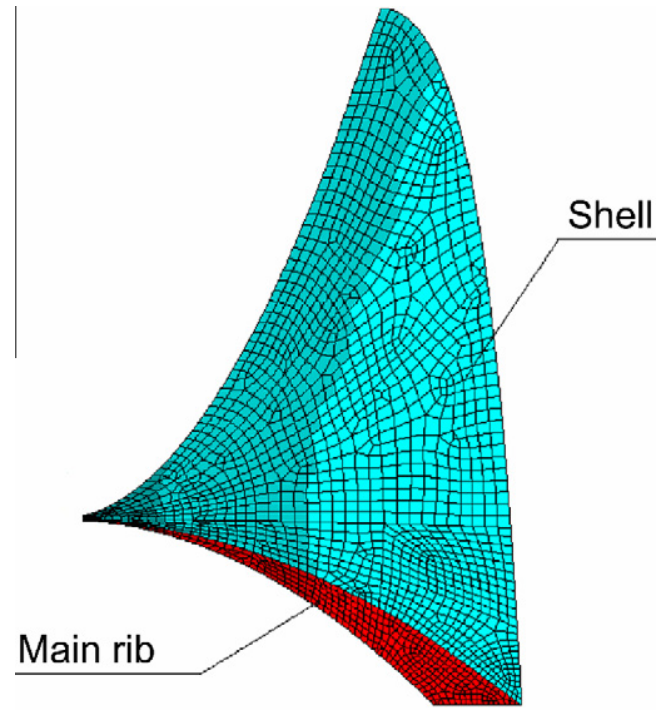

Fig. 8. FE mesh of one sixth of the structure.

a
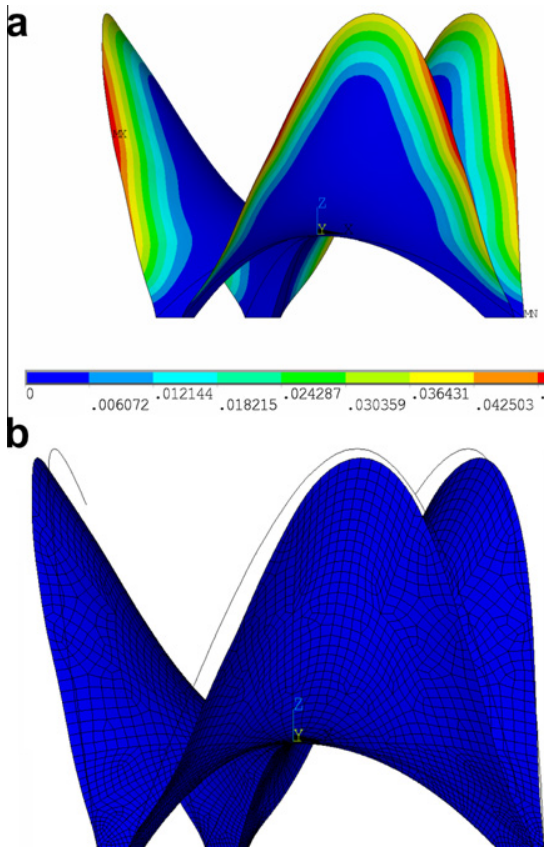

Fig. 9. Hyperbolic paraboloid: (a) displacements, (b) deformed shell.

The outer edge of the spherical cap is cut by cylindrical vaults, which are supported on eight perimeter columns (located at the points of intersection of two cylindrical vaults). To absorb the horizontal forces at the supports, there is an octagonal ring formed by 16 tensioned cables of $0.03 \mathrm{~m}$ diameter. The design of this structure means that meridian forces from the dome, having a direction tangent to the sphere, are transmitted to the columns. The system that is compound by the columns and the perimeter tensioned ring is able to absorb the vertical and horizontal components of the forces in each of the eight supports. The boundary conditions in those eight points consist of restricting the displacements and allowing the rotations.

In the CAD model, a set of keypoints are defined, and are linked by curved lines (splines), leading to a set of arcs to make the areas that form the dome. Specifically, there are 72 keypoints, which generate 75 splines and 28 areas (Fig. 10b).

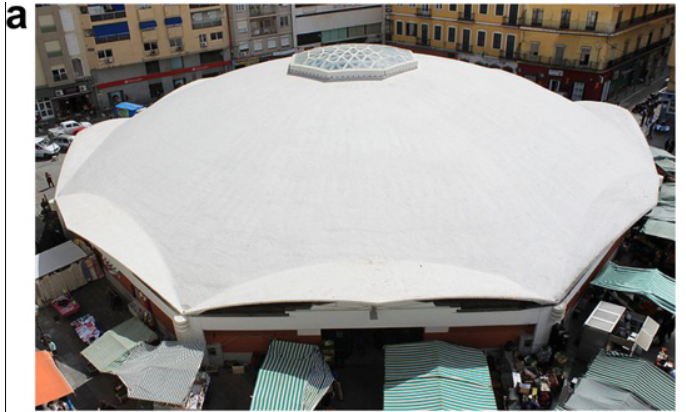

b

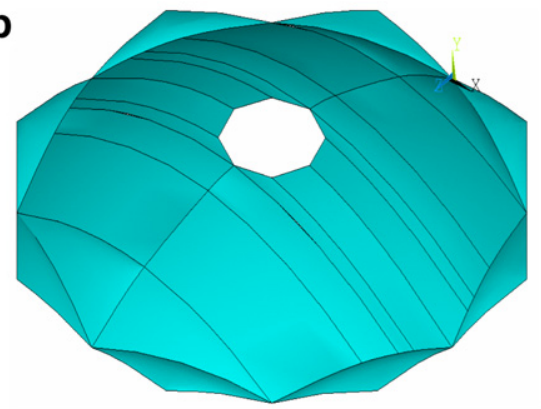

Fig. 10. Dome of Algeciras market: (a) view of the dome [source: www.wikipedia.org], (b) lines and areas of the CAD model.

As in the previous example, the structure is under the gravitational load, so it is sufficient to consider the specific weight of concrete $\left(25 \mathrm{kN} / \mathrm{m}^{3}\right)$.

\subsubsection{Accidental imperfection sensitivity factor $\rho_{\mathrm{hom}}^{*}$. Graphical method}

This method should identify the geometries analysed in Section 3.4 that are closer to the dome of the Algeciras Market. The angle of the dome, measured from the top, is $33^{\circ}$. The geometry that is more similar to the one studied is that of a $36^{\circ}$ angle. In particular, two types of this shell have been analyzed: the one with circular base (Fig. 4c) and that with square base (Fig. 4e), both subject to different support distributions.

As the dome of Algeciras Market weighs on eight columns uniformly distributed at the outer contour, it is consistent to adopt an average value of the accidental imperfection sensitivity factor between the design with hinged supports and that with four supports located at the vertices.

Moreover, as the dome has an octagonal base, it is advisable to compare the results of Fig. $4 \mathrm{c}$ and e. For small values of $w_{0} / e$ (about 0.1 ) the results for $\rho_{\text {hom }}^{*}$ are almost identical. However, upon moving to the right of the graph, the curves are increasingly distant from each other up to a maximum difference of around $30 \%$, and the results for the dome on a square base are more conservative. Specifically, for $w_{0} / e=0.2$ (approximate value for the dome of Algeciras Market), there is a difference of $10 \%$. Given this fact, it is advisable to lessen the average result calculated using Fig. 4e by around $5 \%$, to consider an intermediate case between the dome on a square base and that on a circular base.

Similarly to Section 4.2, the method proposed by Medwadowski (Eq. (6)) has been used. The accidental geometric imperfection $w_{0}^{\prime \prime}$ is $0.01774 \mathrm{~m}$ and the ratio $w_{0}^{\prime \prime} / e$ is 0.20 (considering rigid formwork, $a=1$ ). The results obtained for $\rho_{\text {hom }}^{*}$ in the cases of hinged supports and supports located at the vertices are 0.54 and 1.00 respectively, with the average value being 0.77 , and the reduced value 0.73 .

In the event of not having these graphs for domes, the curve for the sphere and the axially compressed cylinder would have been 
a
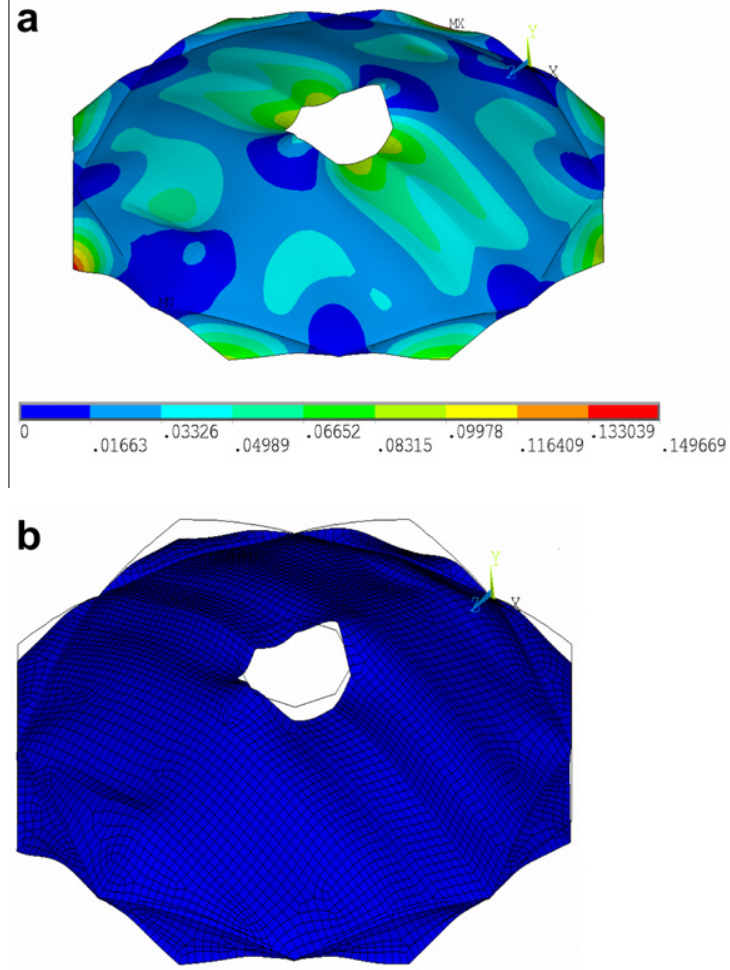

Fig. 11. Dome of Algeciras market: (a) displacements, (b) deformed shell.

Table 1

Geometric parameters of the cooling tower of the Ascó power plant (units in $\mathrm{m}$ ). $a=$ throat radius; $r_{\mathrm{b}}=$ radius at shell lower edge; $r_{\mathrm{t}}=$ radius at the top of the shell; $Z_{\mathrm{t}}=$ vertical ordinate from throat to top of shell; $Z_{\mathrm{b}}=$ vertical ordinate from throat to lower shell edge; $H=$ total height of tower; $t_{\mathrm{h}}=$ shell thickness (variable depending on the height).

\begin{tabular}{lllllll}
\hline$A$ & $r_{\mathrm{b}}$ & $r_{\mathrm{t}}$ & $Z_{\mathrm{t}}$ & $Z_{\mathrm{b}}$ & $H$ & $t_{\mathrm{h}}$ \\
\hline 36.75 & 58.45 & 38.50 & 32.25 & 127.75 & 160.00 & $0.20-0.28$ \\
\hline
\end{tabular}

used, following the IASS Recommendations, so a value of 0.44 would have been obtained, which is considerably more conservative (39.73\% lower).

\subsubsection{Accidental imperfection sensitivity factor $\rho_{\mathrm{hom}}^{*}$. Analytical method}

Calculating similarly to Section 4.2 , the following values are obtained: $p_{\mathrm{cr}}^{\text {lin }}=16.61, p_{\mathrm{cr}}^{\text {upper }}=11.53$ and $\rho_{\mathrm{hom}}^{*}=p_{\mathrm{cr}}^{\text {upper }} / p_{\mathrm{cr}}^{\text {lin }}=0.69$.

Using the graphical method, the factor is $5.48 \%$ more conservative than the analytical result. This error is not significant given that $\rho_{\text {hom }}^{*}$ is corrected by a safety factor, which considers small deviations in the calculation. However, using the IASS Recommendations for spheres, the factor is $36.23 \%$ lower.

\subsubsection{Initial geometric imperfection sensitivity factor $\rho_{\text {hom }}$}

The calculable imperfection $w_{0}^{\prime}$ is quantified by the bending theory of shells. It is necessary to analyse the structure to obtain the deflection just before the start of buckling. Fig. 11 shows the displacements in the dome and the deformed shell. The maximum displacement $w_{0}^{\prime}$ is $0.1497 \mathrm{~m}$.

Using Eq. (4), the global initial geometric imperfection of the shell $w_{0}$ is $0.1674 \mathrm{~m}$ and the ratio $w_{0} / e$ is 1.86 . The ratio $w_{0} / e$ is greater than one, so it runs off the graph. As the trend of the curves is practically constant for higher values of $w_{0} / e$, the value of $\rho_{\text {hom }}$ corresponding to the point $w_{0} / e=1$ in the $w_{0} / e$-axis is considered.
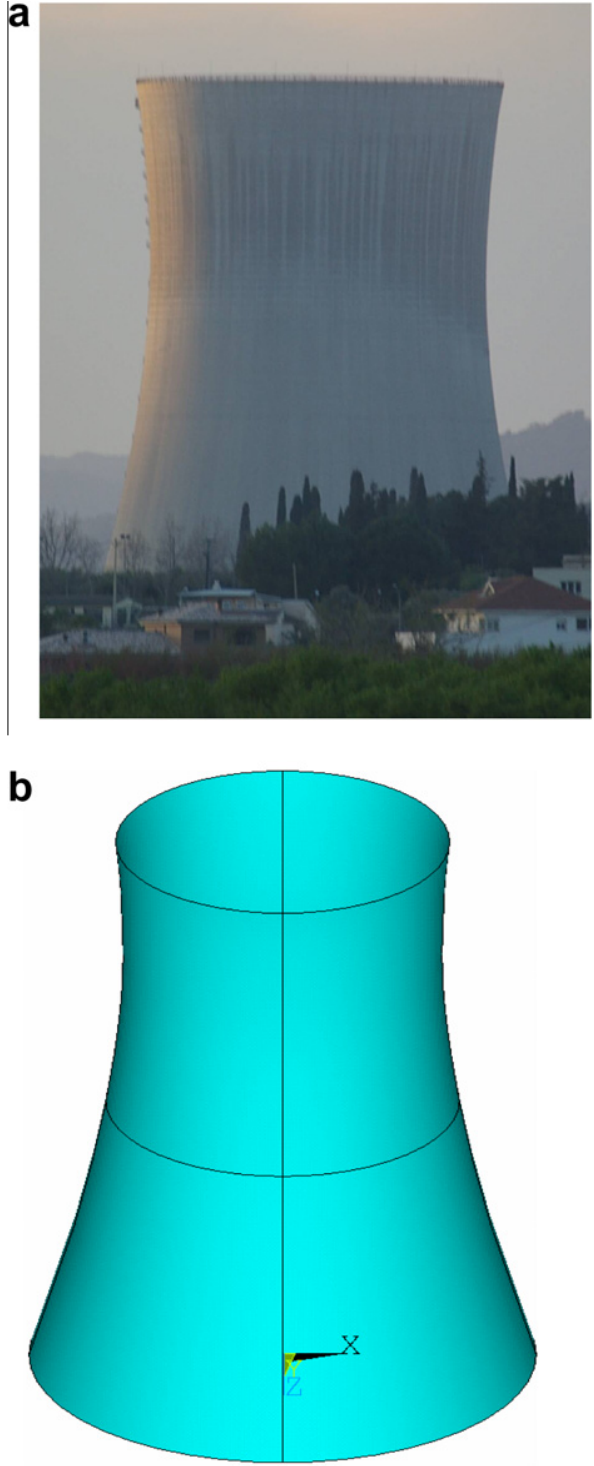

Fig. 12. Cooling tower of the Ascó power plant. (a) View of the tower. [Source: www.wikipedia.org]. (b) Lines and areas of the CAD model.

Using the graphs in Fig. 4c and e, the average value of the initial geometric imperfection sensitivity factor $\rho_{\text {hom }}$, reduced by $5 \%$ to consider that the base of the dome is octagonal, is 0.72 .

Again, an important difference between this factor and that obtained using the curve for spheres is noticed. In the latter case, the factor is $0.20,72.22 \%$ lower than the result obtained with the curves of Fig. $4 \mathrm{c}$ and e.

\subsection{Cooling tower of the Ascó nuclear power plant}

\subsubsection{The hyperbolic rotational surface}

The geometry of the cooling towers corresponds to a hyperbolic rotational surface. The equations that define this type of geometry can be found in $[12,14]$, among others.

The thickness of the cooling towers is variable depending on the height. The IASS Working Group No. 3 [15] proposed to use the equation $\left(R^{2} H\right)^{1 / 3} / 400$ to determine the minimum thickness at different levels, where $R$ is the transverse radius of the tower and $H$ its height.

The values of the geometric parameters that define the cooling tower studied (Fig. 12) are listed in Table 1. 
In the CAD model, there are four keypoints linked by four cylindrical curves at different Z-levels, generating a circumference whose radius depends on the height. These circumferences determine a spline with the shape of the hyperbola that describes the outer surface of the model. Specifically, 28 keypoints are used to generate 32 curves (of which four are splines) and eight areas. The lines and areas that generate the hyperboloid are shown in Fig. 12b.

The cooling tower is supported on columns equidistantly distributed around the base ring. The boundary conditions in these points are zero displacements and free rotations.

As in the previous examples, the structure is under the gravitational load, so it is sufficient to consider the specific weight of the concrete $\left(25 \mathrm{kN} / \mathrm{m}^{3}\right)$.

\subsubsection{Accidental imperfection sensitivity factor $\rho_{\mathrm{hom}}^{*}$. Graphical method}

The principal radii of curvature of this shell are $R_{\theta}=36.75 \mathrm{~m}$ and $R_{\phi}=290.25 \mathrm{~m} \mathrm{[12]}$, and the average radius $R$ is $103.28 \mathrm{~m}$. With this radius and the geometric data shown in Table 1 , a ratio $l^{2} /$ $R e=1377.1$ is calculated. This value is very close to 1000 , which is used to define the medium hyperbolic rotational surfaces studied in Section 3.5. Therefore, the Fig. $5 \mathrm{~d}$ has been used in the calculation of the imperfection sensitivity factor.

It should be noted that the graph used has been obtained for shells with the base ring clamped completely, when in fact the clamped supports are located in some isolated points of the base ring of the tower. A reduction in the stiffness of the support ring implies a reduction in the imperfection sensitivity factor [13].

To account for this situation, a conservative method to quantify the accidental imperfection may be used. The IASS method proposes to consider the same value for the accidental imperfection and for the calculable imperfection, equal to the bending deflection in the shell just before the start of buckling. Fig. 13 shows the displacements in the structure and the deformed shell. The maximum displacement $w_{0}^{\prime}$ is $0.1318 \mathrm{~m}$.

Using the IASS method and Fig. 5d, the accidental geometric imperfection $w_{0}^{\prime \prime}$ is $0.1318 \mathrm{~m}$, and the ratio $w_{0}^{\prime \prime} / e$ is 0.549 . The factor $\rho_{\text {hom }}^{*}$ is 0.58 .

In the event of not having these graphs for rotational hyperboloids, the curve for the sphere and the cylinder axially compressed would have been used, so a value of 0.26 would have been obtained, which is considerably more conservative (55.17\% lower).

\subsubsection{Accidental imperfection sensitivity factor $\rho_{\mathrm{hom}}^{*}$. Analytical method}

Calculating similarly to Sections 4.2 and 4.3 , and adopting an average thickness of the shell of $0.24 \mathrm{~m}$, the following values are obtained: $p_{\mathrm{cr}}^{\text {lin }}=10.49, p_{\mathrm{cr}}^{\text {upper }}=5.77$ and $\rho_{\mathrm{hom}}^{*}=p_{\mathrm{cr}}^{\text {upper }} / p_{\mathrm{cr}}^{\mathrm{lin}}=0.55$.

The result is quite similar to the graphical method, with an error of only $5.17 \%$. As commented before, this error is insignificant because $\rho_{\text {hom }}^{*}$ should be corrected by a safety factor, which considers small deviations in the calculation. However, using the IASS Recommendations for spheres, the factor is $52.73 \%$ lower.

\subsubsection{Initial geometric imperfection sensitivity factor $\rho_{\text {hom }}$}

According the IASS method, the initial geometric imperfection of the hyperbolic tower can be estimated by the addition of the two types of imperfections, resulting $w_{0}=0.2636 \mathrm{~m}$ and the ratio $w_{0} / e=1.10$. This ratio barely exceeds one, so a value of $\rho_{\text {hom }}$ slightly lower than that for $w_{0} / e=1$ is adopted ( $\rho_{\text {hom }}=0.52$ ).

Again, an important difference between this factor and that obtained using the curve for spheres is noticed. In the latter case, the factor is $0.20,61.54 \%$ lower.
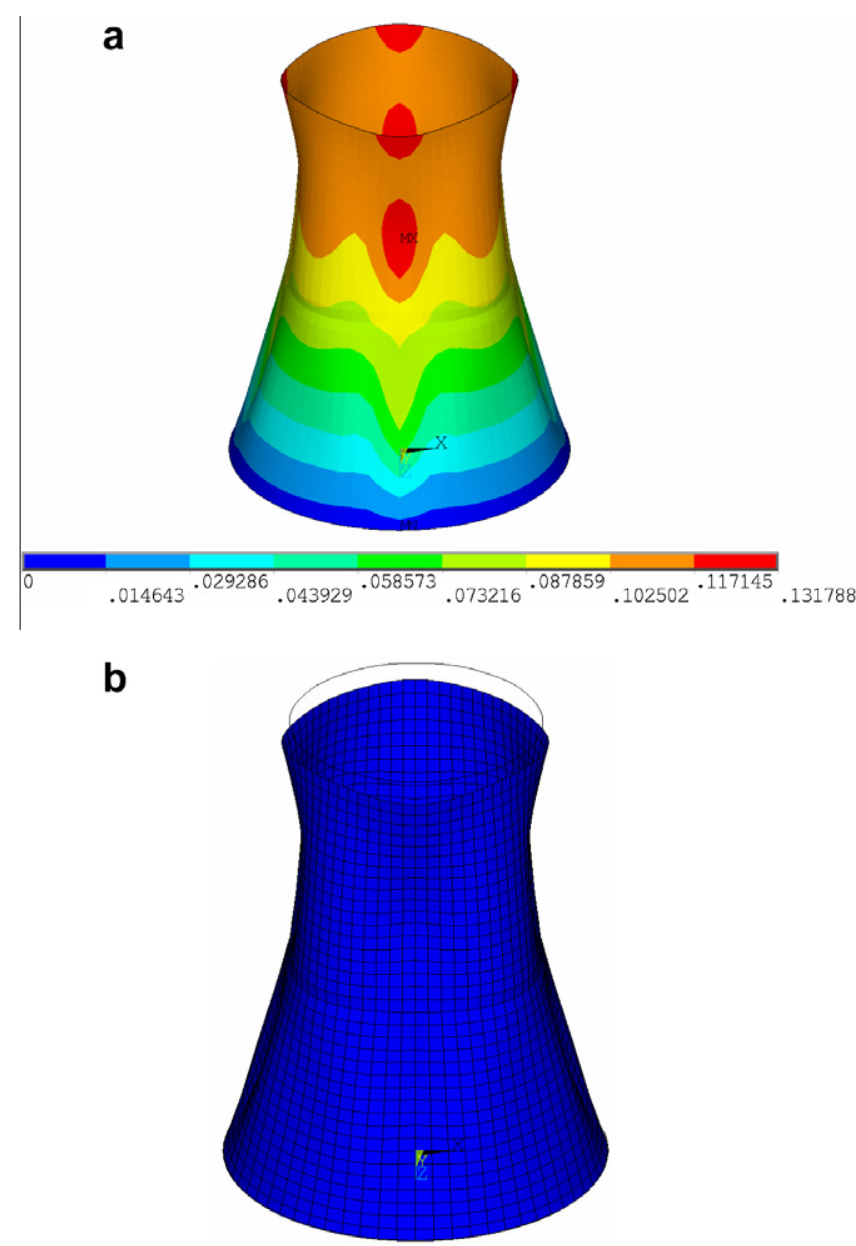

Fig. 13. Cooling tower of the Ascó power plant: (a) displacements, (b) deformed shell.

\section{Conclusions}

In this paper, the influence of imperfection on the buckling load is studied for the case of shells of different geometries (such as spherical dome, barrel vault and double-curvature ruled surface: hyperbolic paraboloid and hyperbolic rotational surface) to those studied in the IASS Recommendations (sphere and cylinder).

The main conclusions of this study can be summarised in the following points:

1. Axially compressed models present the most unfavourable imperfection sensitivity factor, with a trend of the curves similar, or even below those in the IASS Recommendations for spheres and cylinders subjected to axial compression. Most models when laterally compressed, usually adopt intermediate values between the short and the long cylinder, except for the cases of spherical domes.

2. The behaviour of the spherical dome is mainly conditioned by the angle (taken from the ridge). The worst case is the hemisphere (with values slightly higher than the sphere).

3. Shells subjected to gravity show an imperfection sensitivity factor higher than shells subjected to lateral compression (between $5 \%$ and $10 \%$ ).

4. There are no significant differences in the behaviour against imperfection between hinged and clamped supported shells. However, a reduction of the stiffness at the supports such as 
allowing horizontal displacement, presents a significant importance, since it causes a significant reduction in the imperfection sensitivity factor.

5. In general, the less statically indeterminate the configuration of supports is, the closer to one the imperfection sensitivity factor is. However, for a certain geometry of a shell, significant changes in distribution and the number of supports may cause significant changes in imperfection sensitivity.

6. The IASS Recommendation to quantify the influence of geometric imperfection in shells whose behaviour is not known with certainty, by means of the curves for spheres and axially compressed cylinders, is too conservative.

\section{References}

[1] Kollár L, Dulácska E. Buckling of shells for engineers. Chichester: Wiley; 1984

[2] IASS Working Group No. 5. Recommendations for reinforced concrete shells and folded plates. Madrid: IASS, 1979.

[3] Kollár L, Dulácska E. Buckling of shells (in German). Budapest: Akademiai Kiado and Werner-Verlag; 1975

[4] Scordelis AC. Stability of reinforced concrete domes and hyperbolic paraboloidal shells. In: Popov EP, Medwadowski SJ, editors. ACI Special Publication SP-67 Concrete Shells Buckling. Farmington Hills: ACI; 1981.

[5] Medwadowski SJ. Buckling of concrete shells: an overview. J IASS 2004;45(1):51-63.
[6] Kollár L. Buckling of complete spherical shells and spherical caps. In: Buckling of Shells. Berlin: Springer; 1982.

[7] Kollár L. Modified proposal for the text of the recommendations. In: Personal communication to Medwadowski SJ, 1993.

[8] Tomás A. Optimum design of shape and reinforcement for concrete shells (in Spanish). Madrid: ACHE; 2009.

[9] Tomás A, Martí P. Shape and size optimisation of concrete shells. Eng Struct 2010;32(6):1650-8.

[10] Tomás A, Martí P, Tovar JP. Imperfection sensitivity factor in the buckling of single and double curvature concrete shells. In: Topping BHV, Adam JM, Pallarés FJ, Bru R, Romero ML, editors. Proceedings of the tenth international conference on computational structures technology. Civil-Comp Press, Stirlingshire, UK, 2010, Paper 101. doi:10.4203/ccp.93.101.

[11] Dulácska E, Kollár L. Design procedure for the buckling analysis of reinforced concrete shells. Thin-Wall Struct 1995;23(1-4):313-21.

[12] Tovar JP. Influence of initial geometrical imperfections in the buckling of concrete shells (in Spanish). BEng Thesis, Department of Structures and Construction, Technical University of Cartagena (UPCT), Spain, 2009.

[13] Gioncu V. Thin reinforced concrete shells. Chichester: John Wiley \& Sons; 1979.

[14] Gould PL. Analysis of plates and shells. Upper Saddle River: Prentice Hall; 1999.

[15] IASS Working Group No. 3. Recommendations for the design of hyperbolic or other similarly shaped cooling towers. Brussels: IASS; 1977.

[16] Tomás A, Martí P. Optimality of Candela's concrete shells: a study of his posthumous design. J IASS 2010;51(1):67-77.

[17] Domingo A, Lázaro C, Serna P. Design of a thin shell fibre reinforced concrete hypar roof. In: Astudillo R, Madrid AJ, editors. Proceedings of the 40th congress of the IASS. Madrid: CEDEX; 1999.

[18] ANSYS. Advanced analysis techniques guide, Canonsburg: ANSYS Inc, 2004. 